\title{
Low-Profile Fully-Printed Multifrequency Monopoles Loaded with Complementary Metamaterial Transmission Line
}

\author{
Xue $L^{1,2}$, Jinwen TIAN ${ }^{1}$ \\ ${ }^{1}$ Inst. for Pattern Recognition and Artificial Intelligence, Huazhong University of Science and Technology, \\ Wuhan 430074, China \\ ${ }^{2}$ Defense Forces Academy, Zhengzhou 450052, China \\ ghoul_gargoyle@163.com,jwtian@mail.hust.edu.cn
}

\begin{abstract}
The design of a new class of multifrequency monopoles by loading a set of resonant-type complementary metamaterial transmission lines (CMTL) is firstly presented. Two types of CMTL elements are comprehensively explored: the former is the epsilon negative (ENG) one by loading complementary split ring resonators (CSRRs) with different configurations on the signal strip, whereas the latter is the double negative (DNG) one by incorporating the CSRRs and capacitive gaps. In both cases, the CMTLs are considered with different number of unit cells. By cautiously controlling the geometrical parameters of element structure, five antenna prototypes coving different communication standards (GSM, UMTS, $D M B$ and WiMAX) are designed, fabricated and measured. Numerical and experimental results illustrate that the zeroth-order resonance frequencies of the ENG and DNG monopoles are in desirable consistency. Moreover, of all operating frequencies the antennas exhibit fairly good impedance matching performances better than $-10 \mathrm{~dB}$ and quasi-omnidirectional radiation patterns.
\end{abstract}

\section{Keywords}

Metamaterial transmission line, planar monopole, multi-frequency antenna, complementary, zerothorder resonator (ZOR)

\section{Introduction}

Over recent years, there has been a renewed interest in using artificial metamaterial (MTM) transmission line (TL) in the design of microwave devices and components [1]-[20] due to its abnormal electromagnetic (EM) properties that are hardly realized in nature. As to the planar microstrip monopoles [9]-[20], these antennas in general can be classified into three categories according to the loading manner and working mechanism of the MTM TL elements. The monopoles of the first category are based on the double negative (DNG) MTM TLs [9]-[16] which are also termed as composite right/left handed (CRLH) TLs. These antennas can be engineered with broadband or multi-frequency operation because the operating modes can be arbitrarily controlled by the well-conducted dispersion curve of CRLH elements. The second category is the monopole or dipole antennas built on the meta-surface made of periodically arranged left handed (LH) particles [17]. Through this type of loading, the antenna performances are improved in terms of both enhanced radiation behavior and broadened impedance matching bandwidth. The third category is the monopole by introducing LH MTMs [18] or split ring resonators (SRRs) [19] along it or even by embedding complementary SRRs (CSRRs) in the monopoles [20]. In this regard, the miniaturization and band-notch characteristic in an ultrawide operation band were achieved in virtue of the subwavelength resonance of the loaded elements.

Although compactness and multifunction are realized in aforementioned monopoles made of DNG MTM TLs, the mostly reported structures are confined to the nonresonant-type TL elements by using chip components which are restricted to low frequency operation and are not efficient radiators [9]-[12]. As to the rarely reported distributed TL monopoles [13], [14], the shunt inductors are commonly realized by grounded vias which would degrade the antenna gain due to the metallic losses. Moreover, further miniaturization is still a pressing task since the compactness is of great importance to portable and handheld antennas. These issues make an improved and alternative strategy that can be easily characterized and experimentally implemented a pressing task. The goal of this paper is thus to explore a novel avenue in the implementation of fully printed monopoles with simultaneous compact and multifunctional feature. The fundamentals and working mechanism of the monopoles using resonant-type complementary MTM TL (CMTL) will be firstly introduced. Then the multifrequency monopoles made of epsilon negative (ENG) and DNG CMTLs by etching the CSRRs on the signal strip is proposed, characterized and eventually fabricated. 


\section{Resonant-type Microstrip-fed ENG and DNG Monopoles}

\subsection{Fundamentals and Theoretical Background}

The crucial obstacle of pushing the resonant-type CMTL element such as CSRRs in the monopole application is the structure incompatibility because the CSRRs commonly require a ground plane while the host monopole is unable to afford. In this paper, the resonant-type structures utilized for the monopoles are inspired from [16], [21], where the CSRRs are etched on the signal strip for filter and divider applications. Fig. 1 plots the sketch of the conventional and proposed conceptual microstrip-fed monopoles, respectively and the corresponding equivalent circuit model. As a first step towards the design of a DNG monopole, four essential parameters are defined: the phase $\varphi_{C M T L}$ induced by the CMTL element, the phase $\varphi_{\text {Mono }}$ caused by the host monopole, resonant modes (indices) $n$ and the number of utilized CMTL elements $N$. Similar to the nonresonant-type CRLH monopole, the overall possible eigenfrequencies of the DNG monopoles should satisfy the following resonant condition [13], [16]

$$
\varphi_{\text {total }}=N \times \varphi_{\mathrm{CMTL}}+\varphi_{\text {Mono }}=n \pi / 2, n=0, \pm 1, \pm 3, \ldots
$$

where $\varphi_{\text {total }}$ is the phase shift across the entire antenna. Observation from (1) indicates that the relation of the $\varphi_{M o n o}$ and $\varphi_{\text {CMTL }}$ plays an important role in determination of the resonant modes. Note that the stop band of the ENG TL element in the guided wave design can also be employed for a radiated wave design in the case of an open circuit (infinitely large series impedance) or a resonance (often referred to as zeroth-order resonance, ZOR) of the shunt branch in the equivalent circuit model. In both ENG and DNG cases, the current has no place to go except through the shunt branch of the circuit.

\subsection{Monopoles by Loading Single CMTL Element}

Figure 2 shows the flow chart of the formation of the proposed DNG CMTL element evolved from [21]. As can

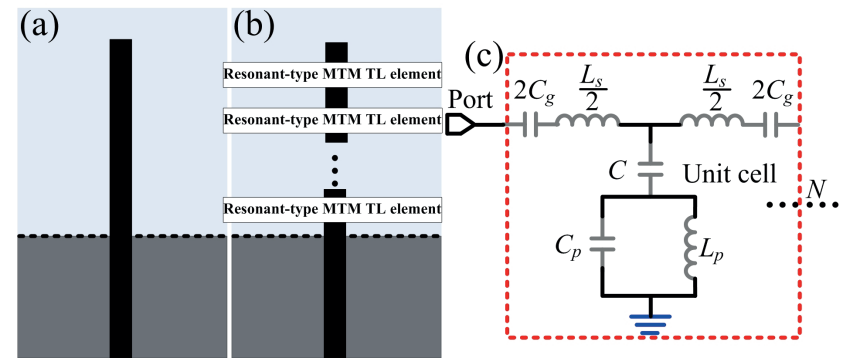

Fig. 1. Sketch of microstrip-fed monopoles: (a) Conventional monopole. (b) Conceptual monopole loaded with resonant-type CMTL elements and (c) equivalent circuit model. be seen, the element is composed of CSRRs etched on the square patch in the upper metallic strip and a square ring with or without two splits in the top and bottom region. The square ring connected to the feedline is formed by introducing an additional gap (closed slot) outside the CSRRs. The CSRRs still response to the axial electric-field component and thus afford the resonant negative permittivity. The resonant effect of the CSRRs is accounted by the parallel tank formed by $L_{p}$ and $C_{p}$ in the circuit model. The capacitive gaps at both sides are modeled by the capacitance $C_{g}$, whereas the inductive effect of the host TL is modeled by the inductance $L_{s}$. Both $C_{g}$ and $L_{s}$ contribute to the negative permeability. The DNG CMTL element can be ENG CMTL element by closing the splits of the square ring (defined as a hybrid ENG resonator) or by removing the gap (CSRRs left only). Since the length of the gap has been extended significantly relative to [21], a larger $C_{g}$ and in turn enhanced LH characteristics will be engineered. In this particular design, since the ground plane of the CMTL element is not placed beneath the CSRRs but is coplanar with the host monopole, the fringing capacitances of the gaps $C_{f}$ are weakened and thus are neglected for convenience. Therefore, the proposed CMTL element can be easily integrated with the planar monopoles of which the ground plane remains unaltered. The $C_{f}$ has nothing to do with the resonant modes but results in slightly reduced resonant frequencies, which will be corroborated by extensive calculations in the upcoming section.

Fig. 3 portrays the layout of the resulting monopoles based on CSRRs-loaded CMTL elements with different configurations. The CMTL element is loaded at the end of

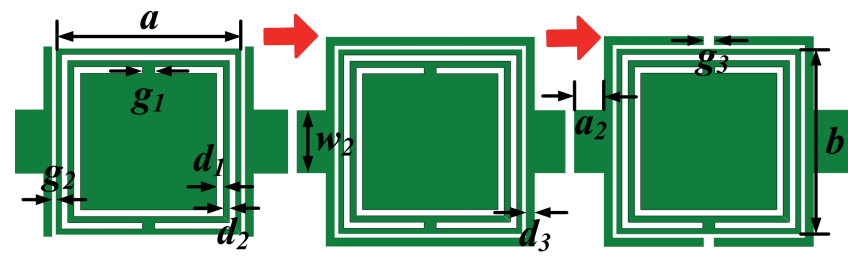

Fig. 2. Flow chart of the formation of the proposed ENG (the middle one) and DNG (the last one) CMTL element evolved from previous CRLH element (the first one) [21].

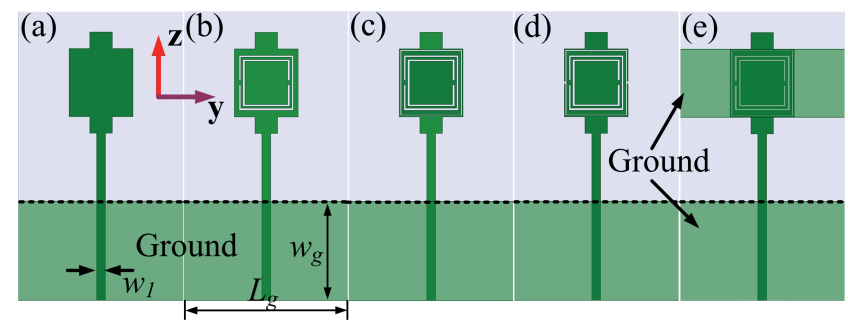

Fig. 3. Layouts of the proposed single-cell microstrip-fed monopoles. (a) Conventional antenna (Case 1); (b) ENG antenna (Case 2); (c) hybrid ENG antenna (Case 3); (d) DNG antenna (Case 4); (e) hybrid ENG antenna with ground underneath the CMTL element (Case 5). The geometrical parameters of these antennas (in millimeter: $\mathrm{mm})$ are $a=11.5, b=10.8 g_{1}=0.72$, $g_{2}=0.2 \quad g_{3}=0.4, \quad d_{1}=d_{2}=0.36, \quad d_{3}=0.2, \quad w_{1}=1.6$, $w_{2}=4, L_{g}=30, w_{g}=18$, and $a_{2}=1.5$. 
the host monopole. A total of six cases are considered for comprehensive analysis. They are orderly the conventional monopole without CMTL loading, the ENG monopoles loaded by the CSRRs only or by aforementioned hybrid resonator, the DNG monopole, the hybrid ENG monopole with a ground plane, and the monopole with the gap only (not shown here for brevity of contents). In the fifth case, the ground beneath the hybrid CMTL element is not connected to that of the host monopole.

The design flow starts from the conventional microstrip-fed monopole where the ground plane $L_{g} \times w_{g}$ is finely designed to obtain a desirable impedance matching over a wide bandwidth and a normal monopolar radiation with high efficiency. The length of the host monopole is designed to operate at GSM band (centered at $1.8 \mathrm{GHz}$ ). All designs are conducted in the commercial full-wave finite-element-method (FEM) EM field simulator Ansoft HFSS (Version of 13.2) and are all layouts are built on the commonly available $1 \mathrm{~mm}$-thick F4B substrate with dielectric constant of $\varepsilon_{r}=2.2$ and loss tangent of $\tan \delta=0.001$. Fig. 4 depicts the simulated reflection coefficients $S_{11}$ of the proposed single-cell MTM-inspired monopoles. To illustrate the effects of the gap, the results of the monopole with the gap only are also provided.

Following Fig. 4, we conclude that the fundamental reflection dip (around GSM $1.8 \mathrm{GHz}$ ) corresponds to the operating frequency $f_{0}$ of the host monopole, whereas the second reflection dip (covering the Satellite Digital Mobile Broadcasting (DMB) band, 2605 $\div 2655 \mathrm{MHz}$ ) corresponds to the ZOR frequency $f_{\mathrm{M} 1}$ of the CSRRs while the third reflection dip (around $4.65 \mathrm{GHz}, f_{\mathrm{S} 2}$ ) corresponds to the resonance of the gap in hybrid DNG antenna case. As previously discussed, $C_{f}$ is negligible when the ground is removed, which finds strong support from the almost constant trend of the reflection response except for the slightly reduced operating frequencies in case 5 due to the enhanced $C_{f}$. A further comparison between case 3 and the case with the gap only also indicates that the CSRRs interact with the gap, leading to the slightly reduced $f_{\mathrm{S} 2}$ in case 3. Moreover, the uniform E-field distributions (not shown for brevity of contents) are clearly observed in CSRRs at $f_{\mathrm{M} 1}$ while are not shown at residual frequencies, indicating a ZOR mode and strong radiation of CSRRs. This feature distinguishes the monopoles from any previous ones by loading chip components which are always not an efficient radiator. The relatively wider bandwidth of the DNG antenna around $f_{\mathrm{M} 1}$ relative to both ENG antennas is due to that the excited $n=+1$ mode is located in close proximity to the $n=0$ mode (ZOR mode). Note that the $n=+1$ mode never occurs in the ENG case. Moreover, the $f_{0}=2.14 \mathrm{GHz}$ (UMTS band) in the DNG antenna case has been shifted upwards. This is because the CMTL element provides less phase shift than conventional monopole. This means that the DNG monopole affords slightly shorter actual length. Most importantly, the ZOR frequency is observed almost the same in all ENG and DNG cases. This is because the ZOR frequency is only dependent on the $L_{p}$ and $C_{p}$ relative to the dimensions of the CSRRs.

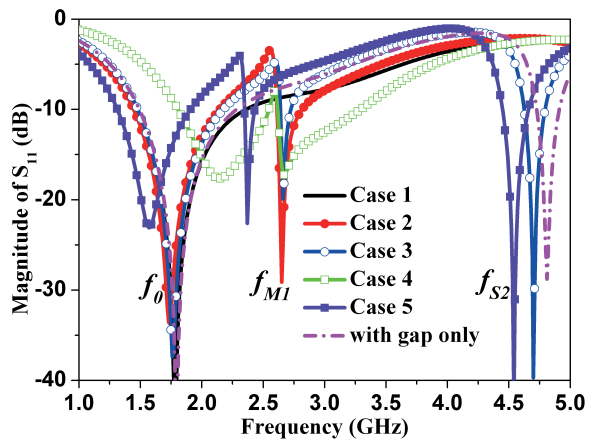

Fig. 4. Simulated reflection coefficients of the proposed single-cell MTM-inspired antennas.

To examine the far-field radiation characteristics, Fig. 5 depicts the 3-D patterns of the MTM-inspired antennas at different frequencies for an intuitionistic view. Following the figure, the monopolar or quasi-monopolar patterns are clearly observed in all cases. The little broken uniformity of the patterns in xoy plane at $f_{\mathrm{S} 2}$ is due to the radiation of the gap which also radiates and thus facilitates the spatial power to be re-synthesized. The detailed performances of these antennas can be referred to Tab. 1. The relatively low efficiency of the ENG antennas at $f_{\mathrm{M} 1}$ with respect to that at $f_{0}$ and $f_{\mathrm{S} 2}$ is due to that $f_{\mathrm{M} 1}$ is located very close to the low efficiency dip (not shown for brevity) at which no radiation occurs. However, it is improved in the DNG case due to double negative permittivity and permeability around $f_{\mathrm{M} 1}$, which enables strong radiation. The low antenna gain in all cases is due to the omnidirectional patterns and small ground dimensions of the host monopole. Further simulation results indicate that the gain is enhanced by an average of $0.7 \mathrm{~dB}$ when $L_{g}$ increases per $10 \mathrm{~mm}$ in some specific range.

For verification, we have fabricated three antenna prototypes (case 2, case 3 and case 4, see orderly in Fig. 6) whose footprints occupy the same area of $1 \times 30 \times 52.6 \mathrm{~mm}^{3}$. Fig. 6 compares the simulated and measured (through a N5230C vector network analyzer) reflection coefficients while Fig. 7 gives the measured radiation patterns in two

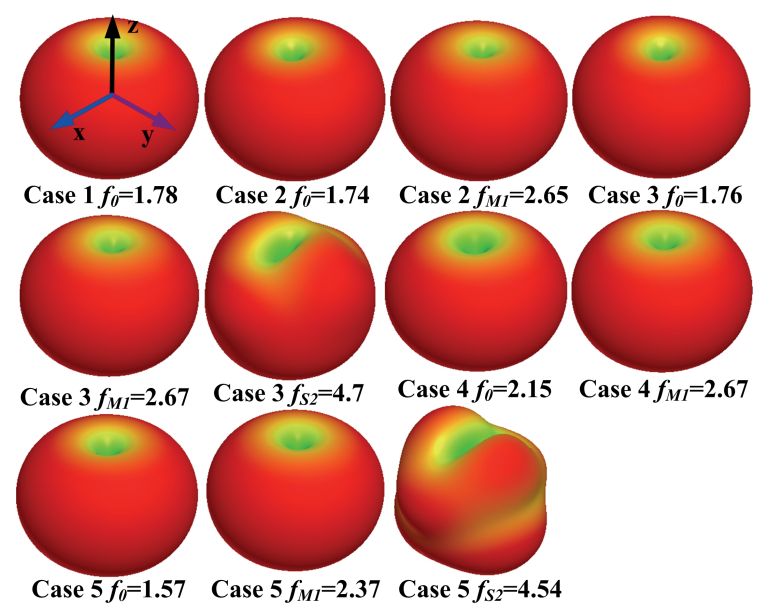

Fig. 5. Simulated 3-D radiation patterns of the single-cell MTM-inspired monopoles at different operating frequencies. 


\begin{tabular}{|c|c|c|c|c|c|c|c|c|c|}
\hline \multirow{2}{*}{ Antennas } & \multicolumn{3}{|c|}{ Antenna gain (dB) } & \multicolumn{3}{|c|}{$\mathrm{BW}(\mathrm{GHz})$} & \multicolumn{3}{|c|}{ Radiation efficiency (\%) } \\
\cline { 2 - 10 } & $f_{0}$ & $f_{\mathrm{M} 1}$ & $f_{\mathrm{S} 2}$ & $f_{0}$ & $f_{\mathrm{M} 1}$ & $f_{\mathrm{S} 2}$ & $f_{0}$ & $f_{\mathrm{M} 1}$ & $f_{\mathrm{S} 2}$ \\
\hline Case 1 & -1.44 & - & - & 0.9 & - & - & 97.5 & - & - \\
\hline Case 2 & -1.64 & 0.59 & - & 0.7 & 0.21 & - & 98.3 & 82.6 & - \\
\hline Case 3 & -1.44 & 0.38 & 0.8 & 0.7 & 0.09 & 0.17 & 97.3 & 75.8 & 89.1 \\
\hline Case 4 & 0.23 & 0.64 & - & 0.75 & 0.67 & - & 95.8 & 88.1 & - \\
\hline
\end{tabular}

Tab. 1. Comparison of performances of the single-cell MTM-inspired monopoles. Note: the bandwidth (BW) is defined by the $-10 \mathrm{~dB}$ reflection coefficients. The efficiency is defined as the radiated power divided by the accepted power at the center frequencies shown in Fig. 5.

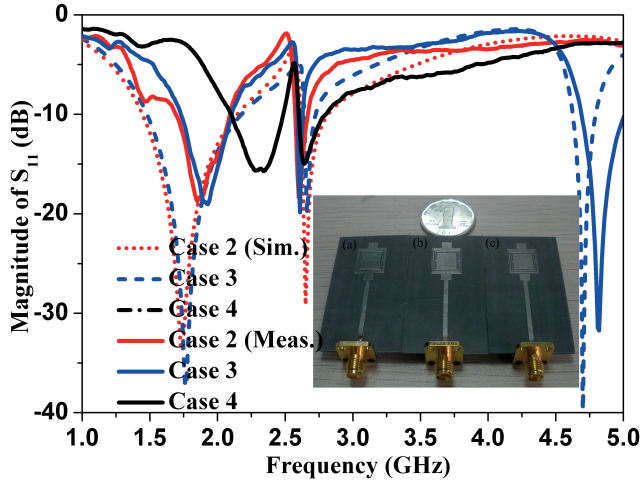

Fig. 6. Simulated and measured reflection coefficients of the fabricated antennas.

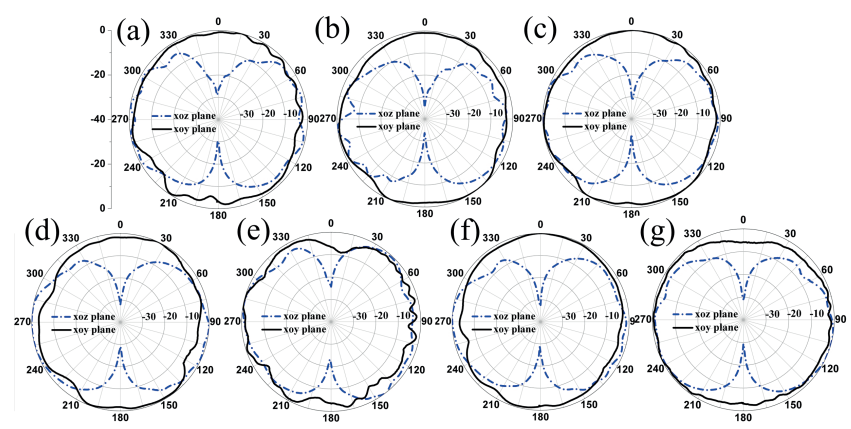

Fig. 7. Measured co-polarized patterns of the (a)-(b) ENG, (c)-(e) hybrid ENG and (f)-(g) DNG antenna at different frequencies. (a) $f_{0}=1.85 \mathrm{GHz}$, (b) $f_{\mathrm{M} 1}=2.6 \mathrm{GHz}$, (c) $f_{0}=1.92 \mathrm{GHz}$, (d) $f_{\mathrm{M} 1}=2.61 \mathrm{GHz}$, (e) $f_{\mathrm{S} 2}=4.81 \mathrm{GHz}$ (f) $f_{0}=2.28 \mathrm{GHz},(\mathrm{g}) f_{\mathrm{M} 1}=2.64 \mathrm{GHz}$

principle planes through a far-field measurement system in an anechoic chamber. As can be seen, a good agreement of results between simulation and measurement is achieved.

The slight frequency upwards in the measurement is attributable to the nonideal substrate that is utilized and also to the tolerances that are inherent in fabrication process. Across all operating frequencies, the measured $S_{11}$ is better than $-10 \mathrm{~dB}$. From Fig. 7, the quasi-monopolar radiations are clearly illustrated at all selected frequencies from the nearly null radiation at the broadside direction in $x o z$ plane while quasi-omnidirectional patterns in xoy plane. Thus far, the multifrequency operation of the proposed antennas by loading single CMTL element has been unambiguously demonstrated.

\subsection{Monopoles by Loading Dual CMTL Elements}

In this section, we will explore the effects of the number of the CMTL elements on the antenna performances. For simplicity, dual CMTL elements are considered without loss of generality. Fig. 8 plots the proposed monopoles by loading dual-cell CMTL elements with the same geometrical parameters and footprints as those shown in Fig. 3. The reflection coefficients are compared in Fig. 9. As can be seen, the impedance matching and bandwidth are almost without deterioration when additional element is loaded for ENG antennas. By contrary, the matching of the DNG antenna deteriorates significantly especially for the not well excited resonance of the host monopole. Nevertheless, the ZOR frequency still exhibits in all ENG and DNG

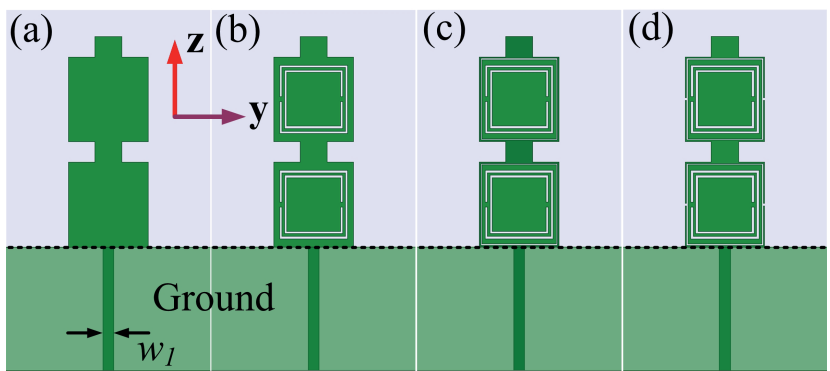

Fig. 8. Layouts of the proposed monopoles by loading dualcell CMTL elements. (a) Conventional antenna; (b) ENG antenna; (c) Hybrid ENG antenna; (d) DNG antenna.

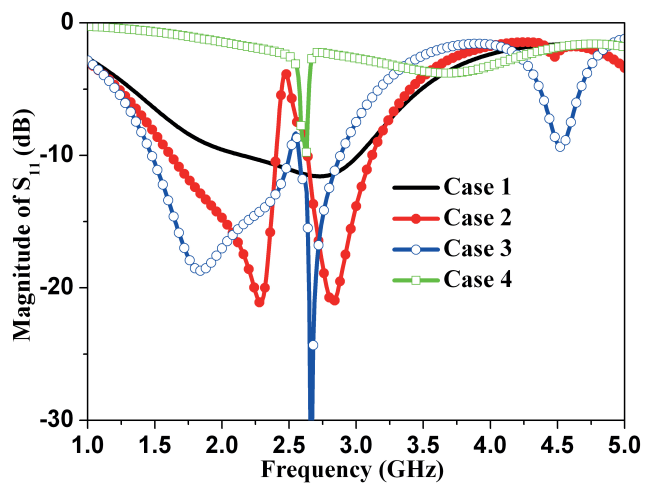

Fig. 9. Simulated reflection coefficients of the proposed dualcell MTM-inspired monopoles. 


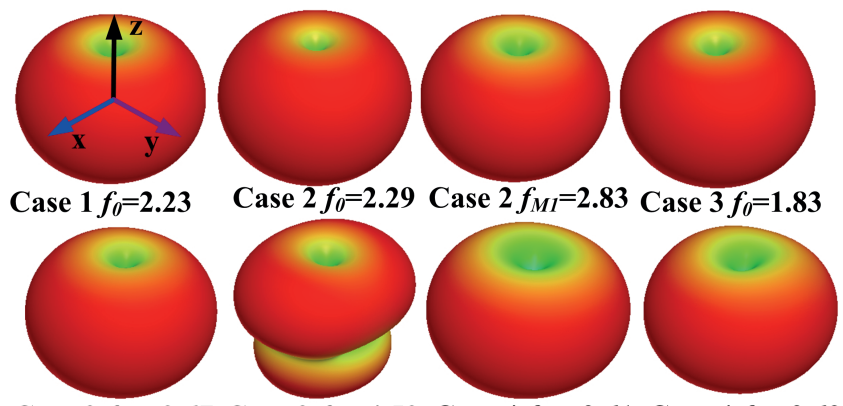

Case $3 f_{M I}=2.67$ Case $3 f_{S 2}=4.53$ Case $4 f_{M I}=2.61$ Case $4 f_{+1}=3.69$

Fig. 10. Simulated 3-D radiation patterns of the dual-cell MTM-inspired monopoles at different operating frequencies.

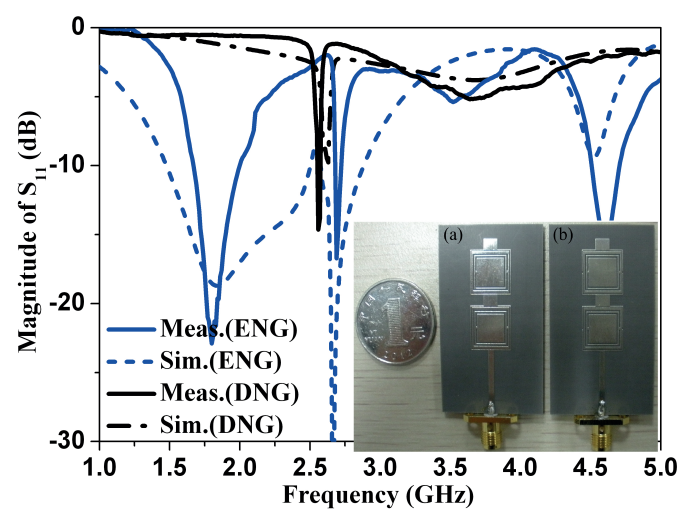

Fig. 11. Simulated and measured reflection coefficients of the dual-cell monopoles.
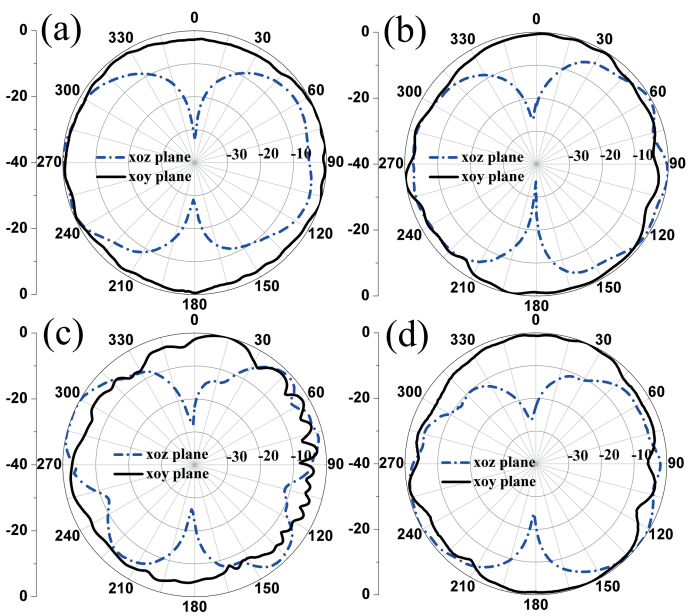

${ }^{0}$ (d)

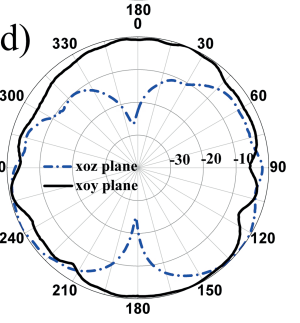

Fig. 12. Measured co-polarized patterns of the (a)-(b) hybrid ENG and (c)-(d) DNG antennas at different frequencies. (a) $f_{0}=1.8 \mathrm{GHz}$, (b) $f_{\mathrm{M} 1}=2.69 \mathrm{GHz}$, (c) $f_{\mathrm{S} 2}=$ $4.61 \mathrm{GHz}$, (d) $f_{0}=2.56 \mathrm{GHz}$.

antennas around $2.6 \mathrm{GHz}$, further demonstrating that ZOR mode is independent while the input impedance is seriously dependent on the number of MTM elements [22]. In DNG case, the $n=+1$ mode around $3.5 \mathrm{GHz}$ (WiMAX band) is weakly excited. Fig. 10 gives the 3-D far-field radiation patterns. Almost the same phenomena are expected as those shown in Fig. 5 except for the antenna gain which has been considerably improved when additional CMTL element is loaded. The reason is because that the CSRRs contribute to the major radiation of the monopole as previously discussed.

For verification, the hybrid ENG and DNG antennas are fabricated. Fig. 11 plots the comparison of reflection coefficients between simulations and measurements while Fig. 12 depicts the measured radiation patterns in two principle planes. A desirable agreement of results is also observed. The reason for slight deviations especially for the narrower bandwidth in the measurement case is due to that the CMTL dimensions of prototypes deviate from those in the simulation model. Nevertheless, the measured $S_{11}$ is better than $-10 \mathrm{~dB}$ over all operating frequencies. From Fig. 12, the measured typical monopolar patterns further confirm the effectiveness of dual-cell elements in the monopole design.

\section{Conclusion}

The use of resonant-type ENG and DNG CMTLs in the design of low-profile multifrequency monopoles is presented. Results reveal that at all operating frequencies the monopoles exhibit quasi-monopolar radiation patterns and fairy good impedance matching performances with return loss better than $10 \mathrm{~dB}$. Moreover, the ZOR frequency is independent on the number of unit cells and is observed as the same for both ENG and DNG antennas. These antennas feature compact, low profile, completely uniplanar, and are without any metallic vias and lumped loadings, rendering easy avenue toward monopoles with multifunction and high integration by using simple photolithography. Other variations of CSRRs and geometrical parameters can be explored for arbitrary manipulation of frequency ratio and further improvement of performances.

\section{References}

[1] ELEFTHERIADES, G. V., BALMAIN, K. G. Negative Refraction Metamaterials: Fundamental Principles and Applications. Hoboken, NJ: Wiley, 2005.

[2] XU, H.-X., WANG, G.-M., LIANG, J.-G., PENG, Q. Novel CRLH TL based on fractal geometry and series power divider application. Acta Physica Sinica, 2012, vol. 61, no. 7, p. 074101.

[3] XU, H.-X., WANG, G.-M., LIANG, J.-G. Novel designed CSRRs and its application in tunable tri-band bandpass filter based on fractal geometry. Radioengineering, 2011, vol. 20, no. 1, p. 312 to 316.

[4] CALOZ, C., ITOH, T. Electromagnetic Metamaterials: Transmission Line Theory and Microwave Applications: The Engineering Approach. Hoboken, NJ: Wiley, 2006.

[5] XU, H.-X., WANG, G.-M., ZHANG, C.-X., WANG, X. Characterization of composite right/left handed transmission line. Electronics Letters, 2011, vol. 47, no. 18, p. 1030-1032. DOI: 10.1049/el.2010.3707

[6] MARQUES, R., MARTIN, F., SOROLLA, M. Metamaterials with Negative Parameters: Theory, Design, and Microwave Applications. Hoboken, NJ: Wiley, 2008. 
[7] XU, H.-X., WANG, G.-M., ZHANG, C.-X., LIANG, J.-G. Novel design of compact microstrip diplexer based on fractal-shaped composite right/left handed transmission line. Journal of Infrared and Millimeter Waves, 2011, vol. 30, no. 5, p. 390-396. DOI: 10.3724/sp.j.1010.2011.00390

[8] XU, H.-X., WANG, G.-M., CHEN, X., LI, T.-P. Broadband balun using fully artificial fractal-shaped composite right/left handed transmission line. IEEE Microwave and Wireless Components Letters, 2012, vol. 22, no. 1, p. 16-18. DOI: 10.1109/LMWC.2011.2173929

[9] KIM, D., KIM, M. Narrow-beamwidth T-shaped monopole antenna fabricated from metamaterial wires. Electronics Letters, 2008, vol. 44, no. 3, p. 180-182. DOI: 10.1049/el:20082854

[10] ANTONiades, M. A., EleftheriadeS, G. V. A foldedmonopole model for electrically small NRI-TL metamaterial antennas. IEEE Antennas and Wireless Propagation Letters, 2008, vol. 7, p. 425-428. DOI: 10.1109/LAWP.2008.2008773

[11] JI, J. K., KIM, G. H., SEONG, W. M. Bandwidth enhancement of metamaterial antennas based on composite right/left-handed transmission line. IEEE Antennas and Wireless Propagation Letters, 2010, vol. 9, p. 36-39. DOI: 10.1109/LAWP.2010.2041628

[12] ZHU, J., ANTONIADES, M. A., ELEFTHERIADES, G. V. A compact tri-band monopole antenna with single-cell metamaterial loading. IEEE Transactions on Antennas and Propagation, 2010, vol. 58, no. 4, p. 1031-1038. DOI: 10.1109/TAP.2010.2041317

[13] IBRAHIM, A., SAFWAT, A. M. E., EL-HENNAWY, H. Tripleband microstrip-fed monopole antenna loaded with CRLH unit cell. IEEE Antennas and Wireless Propagation Letters, 2011, vol. 10, p. 1547-1550. DOI: 10.1109/LAWP.2011.2181813

[14] ANTONIADES, M. A., ElEFTHERIADES, G. V., ROGERS, E. $\mathrm{S}$. A broadband dual-mode monopole antenna using NRI-TL metamaterial loading, IEEE Antennas and Wireless Propagation Letters, 2009, vol. 8, p. 258-261. DOI:10.1109/LAWP.2009.2014402

[15] KOKKINOS, T., FERESIDIS, A. P. Low-profile folded monopoles with embedded planar metamaterial phase-shifting lines. IEEE Transactions on Antennas and Propagation, 2009, vol. 57, p. 2997-3008. DOI: 10.1109/TAP.2009.2028605

[16] XU, H.-X., WANG, G.-M., LV, Y.-Y., QI, M.-Q., GAO, X., GE, S Multifrequency monopole antennas by loading metamaterial transmission lines with dual-shunt branch circuit. Progress in Electromagnetics Research, 2013, vol. 137, p. 703-725.

[17] ELSHEAK, D. N., ISKANDER, M. F., ELSADE, H. A., ABDALLAH, E. A., ELHENAWY, H. Enhancement of ultra-wideband microstrip monopole antenna by using unequal arms V-shaped slot printed on metamaterial surface. Microwave and Optical
Technology Letters, 2010, vol. 52, no. 10, p. 2203-2209. DOI: 10.1002/mop. 25447

[18] PALANDOKEN, M., GREDE, A., HENKE, H. Broadband microstrip antenna with left-handed metamaterials. IEEE Transactions on Antennas and Propagation, 2009, vol. 57, no. 2, p. 331-338. DOI: 10.1109/TAP.2008.2011230

[19] BARBUTO, M., BILOTTI, F., TOSCANO, A. Design of a multifunctional SRR-loaded printed monopole antenna. International Journal of RF and Microwave Computer-Aided Engineering, 2012, vol. 22, no. 4, p. 552-557. DOI: $10.1002 /$ mmce. 20645

[20] LIU, J., GONG, S., XU, Y., ZHANG, X., FENG, C., QI, N. Compact printed ultra-wideband monopole antenna with dual band-notched characteristics. Electronics Letters, 2008, vol. 44, no. 12 , p. 710-711. DOI: 10.1049/el:20080931

[21] GIL, M., BONACHE, J., MARTIN, F. Synthesis and applications of new left handed microstrip lines with complementary split-ring resonators etched on the signal strip. IET Microwaves Antennas and Propagation, 2008, vol. 2, no. 4, p. 324-330. DOI:10.1049/iet-map:20070225

[22] XU, H.-X., WANG, G.-M., GONG, J.-Q. Compact dual-band zeroth-order resonance antenna. Chinese Physics Letters, 2012, vol. 29, no.1, p. 014101-1-014101-4. DOI: 10.1088/0256$307 \mathrm{X} / 29 / 1 / 014101$

\section{About the Authors ...}

Xue LI was born in Henan province of China. He received his MS degree from the Ordnance Engineering University, Shijiazhuang, China, in 2003, and MS degree from the PLA Information Engineering University, Zhengzhou, China, in 2006. He is now pursing his $\mathrm{PhD}$ degree. His research interests include computer vision and pattern recognition.

Jinwen TIAN was born in the Hebei province of China. $\mathrm{He}$ received his BS degree from the Daqing Petroleum Institute, Daqing, China, in 1983, and MS and PhD degrees from Huazhong University of Science and Technology, Wuhan, China, in 1994 and 1998, respectively. His current interest includes remote sensing image information processing, streaming media technology and its applications, the wavelet transform theory and its applications, image data compression, target detection and recognition, augmented reality and computer software simulation, etc. 